\title{
PTCH1 Mutation in a Patient With Metastatic Undifferentiated Carcinoma With Clear Cell Change
}

\author{
Katy K. Tsai, MDa; Neharika Khurana, MS ${ }^{b}$; Timothy McCalmont, MD,d; Adil Daud, MDa;
} Boris Bastian, MD, $\mathrm{PhD}^{\mathrm{c}, \mathrm{d}}$; and Iwei Yeh, MD, $\mathrm{PhD}^{\mathrm{c}, \mathrm{d}}$

\begin{abstract}
Clear cell basal cell carcinoma (BCC) is an unusual variant of BCC. Its pathogenesis, prognosis, and optimal management remain poorly described due to its rarity. This report presents a 51 -year-old man with a history of excised BCC and cutaneous squamous cell carcinomas of the face, with multiple recurrent poorly differentiated carcinomas with clear cell changes of the shoulder for which further classification using conventional histologic means was not possible. His tumor tissue was sent to Foundation Medicine for testing, which revealed a high number of pathogenic genomic alterations, including a mutation in PTCH1. He was diagnosed with dedifferentiated BCC and started on vismodegib. He developed lung metastases while receiving vismodegib, and his disease continued to progress while he was undergoing treatment in a phase I clinical trial. Given the high number of pathogenic alterations suggestive of high tumor mutational burden, immunotherapy was considered and off-label authorization was obtained for treatment with a PD-1 antibody (pembrolizumab). He had a dramatic disease response after 4 infusions of pembrolizumab. Molecular testing was instrumental in determining the correct diagnosis and formulating appropriate treatment options for this patient. Molecular profiling of metastatic BCCs and its subtypes is essential to the development of effective targeted therapies and combination approaches.
\end{abstract}

J Natl Compr Canc Netw 2019;17(7):778-783 doi: 10.6004/jnccn.2019.7313

a Melanoma and Skin Cancer Program, UCSF Helen Diller Family Comprehensive Cancer Center, San Francisco, California; ${ }^{\circ}$ Oschsner Clinical School, University of Queensland, New Orleans, Louisiana; and 'Department of Dermatology, and dDepartment of Pathology, UCSF Helen Diller Family Comprehensive Cancer Center, San Francisco, California.
Cutaneous neoplasms have a broad spectrum of morphologic presentations. Neoplasms with clear cell morphology, defined as cells with optically clear cytoplasm caused by lipid, glycogen, or mucin accumulation, have been well described and are comprised of primary or metastatic lesions with epidermal, adnexal, mesenchymal, or melanocytic differentiation. ${ }^{1,2}$ Because clear cell morphology is easily identified but nonspecific, ancillary studies such as immunohistochemical staining or molecular studies are helpful in establishing the histogenesis of these tumors.

Clear cell change has been described in epidermal tumors, including acanthoma, seborrheic keratosis, squamous cell carcinoma (SCC), and basal cell carcinoma (BCC). BCC is the most common cancer in the United States, with $>2$ million cases occurring annually. ${ }^{3}$ Although known for its ability to cause significant local destruction of soft tissue, cartilage, and bone, it often has a good prognosis because of its low metastatic rate of $<0.1 \%{ }^{4-6}$ The pathogenesis of BCC has also been well characterized, with the sonic hedgehog (Shh) signaling pathway identified as playing a crucial role. Signaling in this pathway is initiated by the cell surface receptor smoothened homolog (SMO). This pathway is normally inhibited by the cell surface receptor PTCH1, and binding of the hedgehog ligand to PTCH1 prevents this inhibition. The $\mathrm{PTCH}$ gene on chromosome $9 q$ codes for the Shh receptor, and mutations in this gene result in loss of inhibition of SMO activation of the hedgehog pathway and are drivers of nevoid BCC syndrome and $30 \%$ to $90 \%$ of sporadically occurring BCCs. ${ }^{7-9}$ Ultraviolet-induced mutations in the tumor suppressor gene TP53 have also been implicated in BCC development. ${ }^{10}$

In contrast, clear cell BCC is rare. It was first described in $1984,{ }^{11}$ and to date only approximately 30 cases have been described in the literature. These cases, however, have focused on histopathologic/immunohistochemical characteristics of resected primary clear cell BCCs. One

See page 886 for related commentary. 
case report describes clear cell BCC with oligometastatic disease ${ }^{12}$ but molecular profiling was not performed and disease outcome is unknown; recurrence occurred after surgery, but no description of subsequent systemic therapy was provided. The molecular profile and clinical behavior of clear cell BCC thus remains poorly described.

Accordingly, we report a case of undifferentiated carcinoma with clear cell change. Identification of a PTCH1 mutation through molecular profiling was instrumental in correctly diagnosing clear cell BCC and in identifying rational treatment options for metastatic disease.

\section{Case Report}

A 51-year-old man presented to the UCSF Melanoma and Skin Cancer Program in June 2015 with a rapidly enlarging mass of the right trapezius. He described a 10 pack-year smoking history and previous BCC and SCC excision from the left postauricular region in 2005. His recent history was also notable for growth of a right upper back tumor in February 2015, which was resected in late April 2015, with pathology indicating poorly differentiated clear cell carcinoma (Figure 1). The tumor was $9.8 \mathrm{~cm}$ in greatest diameter and was excised with a positive margin. Focal perineural invasion and vaguely squamoid areas were also noted. No adjuvant therapy was administered. On clinical examination at his June 2015 visit, the patient had soft tissue swelling over his right trapezius with ipsilateral supraclavicular lymphadenopathy. PET/CT performed on July 10, 2015, confirmed the presence of a $4.8-\mathrm{cm}$ mass (maximum standardized uptake value $\left.\left[\mathrm{SUV}_{\max }\right], 17.7\right)$ in the right supraclavicular region extending to the posterior neck, with an ipsilateral trapezius satellite nodule $\left(\mathrm{SUV}_{\max }, 8.2\right)$. A hiatal hernia was noted, but no distant metastatic disease was seen.

Review of the patient's external records revealed an extensive oncologic history. After the BCC and SCC excisions from his left postauricular region in 2005, he developed a nonhealing ulcer of the left mandibular angle in 2009 that was diagnosed as BCC and for which he underwent 3 weeks of radiation followed by surgery with wide excision, left superficial parotidectomy, and left modified neck dissection with a left pectoralis rotation flap.

In 2013, the patient had 2 recurrences at the left neck. In February 2013, biopsy of a left neck lesion showed BCC, for which he underwent wide excision with negative margins and a split-thickness skin graft. In July 2013, a new lesion appeared within the graft. Biopsy again showed BCC, and he underwent surgical excision, with an intraoperative finding of a deeper separate tumor. Final pathology noted a poorly differentiated clear cell carcinoma. After undergoing postoperative radiation to the left neck, he did well until
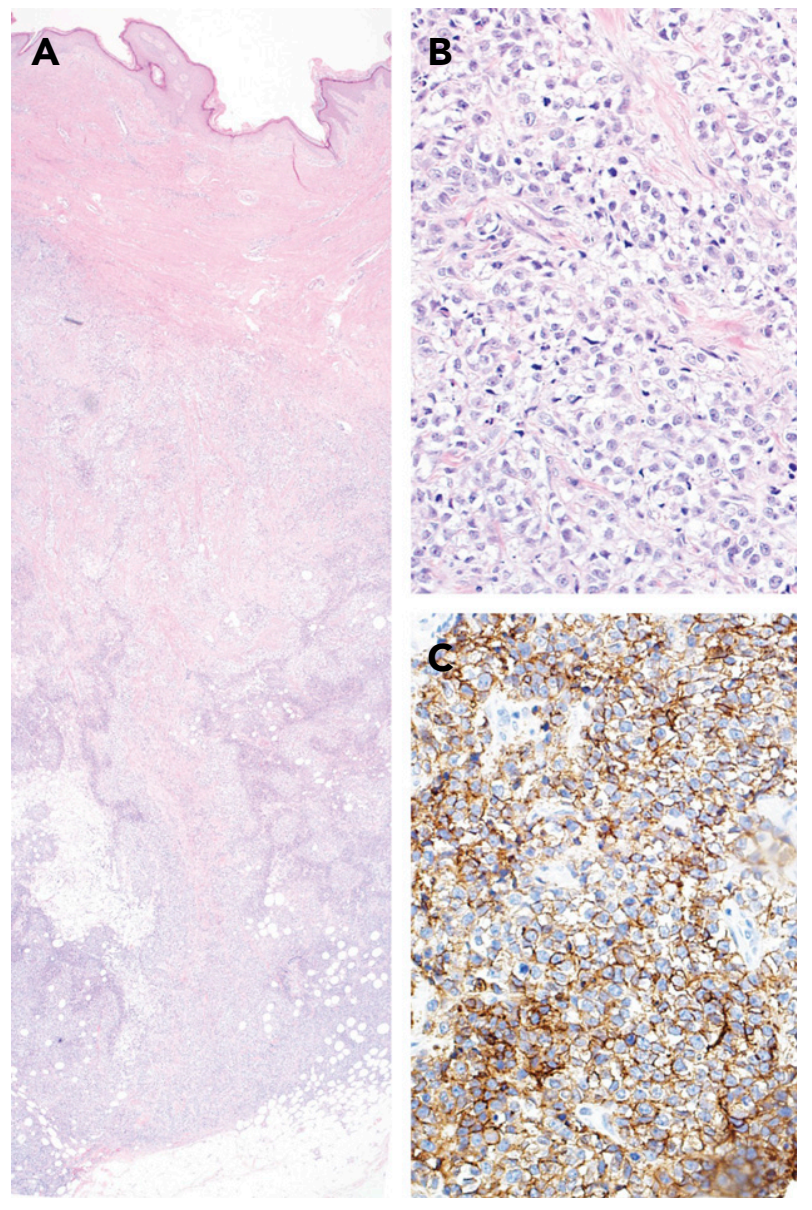

Figure 1. Dermatopathology images showing (A) low-power overview of clear cell carcinoma extending to the subcutis (hematoxylin and esoin, original magnification $\times 4$ ); (B) high-power view of clear cell carcinoma (hematoxylin and eosin, original magnification $\times 10$ ), and (C) high-power view of a BerEP4 immunohistochemical stain showing BerEP4 expression by neoplastic keratinocytes (original magnification $\times 10$ ).

February 2015, when he noticed the right upper back mass for which he underwent surgery in April 2015.

Surgery was not immediately recommended after results of the July 2015 PET/CT scan noted the masses in the right trapezius and supraclavicular region. This case was presented at a multidisciplinary tumor board. Although the disease was clearly a metastatic poorly differentiated carcinoma with clear cell change, further classification was needed to formulate an appropriate treatment plan. The differential diagnosis included BCC, SCC, or clear cell carcinoma of adnexal origin, all of which would have been treated differently from a clinical standpoint. Further classification through conventional pathologic means was not believed possible, and thus the tumor board recommended tumor molecular profiling to help with diagnosis and identification of possible therapeutic options. A formalin-fixed, paraffin-embedded tumor sample was 
submitted to Foundation Medicine for comprehensive genomic profiling. FoundationOne is a next-generation sequencing-based assay developed and validated to identify genomic alterations within hundreds of cancer-related genes. ${ }^{13}$ At the time of this patient's tissue submission, the assay was noted to interrogate 315 genes and introns of 28 genes involved in rearrangements.

On August 11, 2015, the FoundationOne results noted many pathogenic genomic alterations (Table 1). Of particular interest were 2 disabling mutations in PTCH1: one frameshift and the other truncating. Also noted was an inactivating mutation in $M L H 1$, an alteration that results in defective DNA mismatch repair (dMMR) and can result in microsatellite instability (MSI) in a variety of solid tumors. Given the known role of $\mathrm{PTCH} 1$ gene mutation and the Shh signaling pathway in BCC pathogenesis, the patient was diagnosed with clear cell BCC and was started on vismodegib on August 28, 2015. Although he tolerated treatment well, he experienced increasing discomfort and limited range of motion in his right upper extremity. The right trapezius mass continued to enlarge, and subsequent PET/CT performed on October 28,2015 , confirmed progressive disease characterized by a larger and more avid right trapezius mass $(2.2 \mathrm{~cm}$; $\left.\mathrm{SUV}_{\max }, 22.3\right)$, new right axillary lymphadenopathy $\left(1.6 \mathrm{~cm}\right.$; $\left.\mathrm{SUV}_{\max }, 13.8\right)$, and new pulmonary nodules in the right upper lobe $\left(1 \mathrm{~cm}\right.$; $\left.\mathrm{SUV}_{\max }, 6.2\right)$ and lingula $\left(0.8 \mathrm{~cm}\right.$; $\left.\mathrm{SUV}_{\max }, 3.5\right)$. Vismodegib was discontinued on December 28, 2015, and he was switched to sonidegib while clinical trial options were considered. Alternatives to trial participation were discussed, including off-label therapies based on other alterations (ie, MYCN, MLH1, PIK3R1, ARIDIA, and TSC1) and continuing sonidegib, but the patient was ultimately enrolled in a phase I study of an investigational agent starting February 23, 2016.

Restaging scans obtained on April 14, 2016, showed progressive disease, and he was withdrawn from the trial. Enlarged masses in the right trapezius $(10.7 \mathrm{~cm})$, lingula $(2.8 \mathrm{~cm})$, and right axilla $(3.3 \mathrm{~cm})$ were noted. Other therapies, including molecularly targeted therapy and immune checkpoint blockade, were considered. The PD-L1 IHC 28-8 pharmDx assay by NeoGenomics Laboratories stained $0 \%$ of tumor; however, in light of the high number of pathogenic alterations (22 genomic alterations in 18 genes; Table 1) and the documentation of an inactivating MLH-1 alteration suggestive of dMMR/MSI-high status, immunotherapy was believed to be a viable option. Off-label authorization was obtained for a PD-1 antibody (pembrolizumab) and treatment was started on May 9, 2016. The treatment course was notable for grade 1 fatigue and dramatic reduction of the right trapezius mass (Figure 2), with

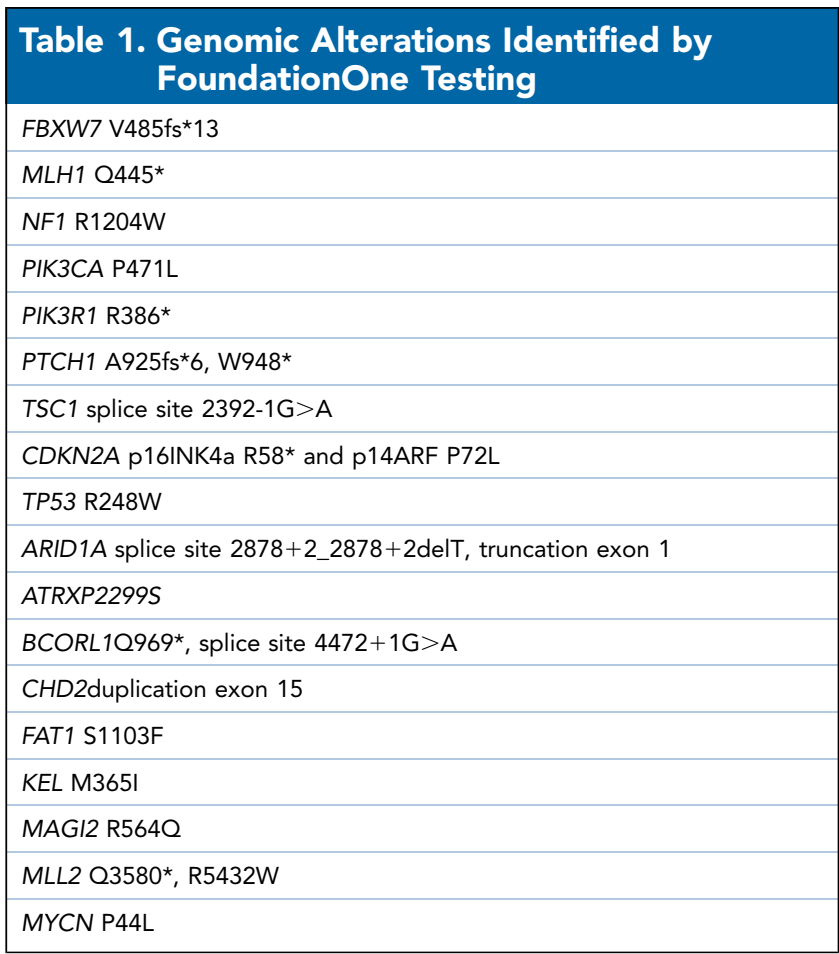

systemic response confirmed on PET/CT scans after 4 infusions (Figure 3 ). The patient received his last infusion on July 24, 2017, after scans confirmed no evidence of disease, and as of February 2018, he remained disease-free.

\section{Discussion}

Molecular profiling is important in resolving diagnostic quandaries and also in leading the way to therapeutic opportunities for rare diseases. This has been shown in the evolution of systemic treatment of unresectable BCC from cytotoxic chemotherapy to a molecularly targeted approach. Chemotherapy has shown some activity in case reports and small series, but advances in understanding BCC genetics and the role of the Shh signaling pathway have allowed for more focused treatment strategies. Currently, 2 hedgehog pathway inhibitors are approved for use in patients with locally advanced BCC that is ineligible for or has recurred after surgery/ radiation: vismodegib and sonidegib. Vismodegib was FDA-approved in 2012 based on promising data from ERIVANCE, a multisite, single-arm, phase II trial of 104 patients. ${ }^{14}$ Subsequent analyses have shown durable responses and consistent efficacy and safety profiles, with an objective response rate (ORR) of $48.5 \%$ and median progression-free survival (PFS) of 9.3 months in patients with metastatic BCC. ${ }^{15,16}$ A subsequent, larger phase II trial (STEVIE) of 1,161 patients with locally advanced or metastatic BCC showed an ORR of 
$5 / 9 / 16$

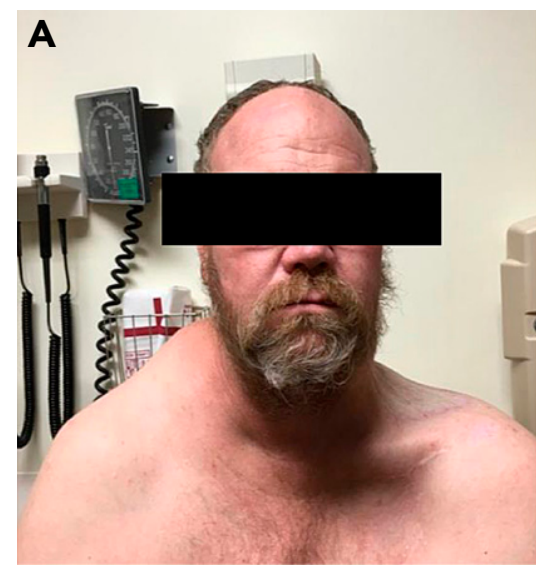

$8 / 1 / 16$

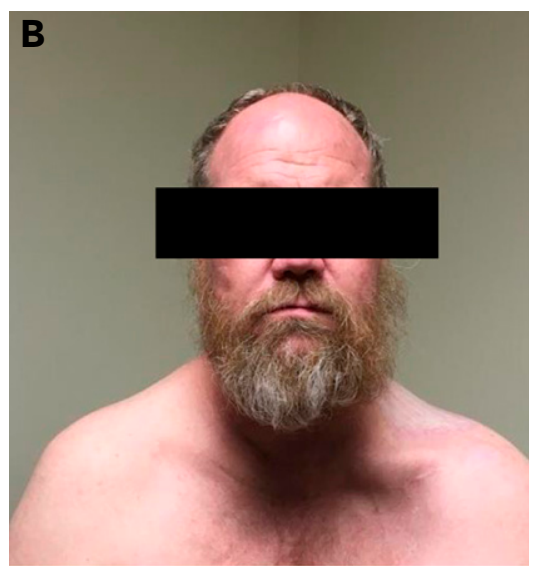

$2 / 22 / 18$

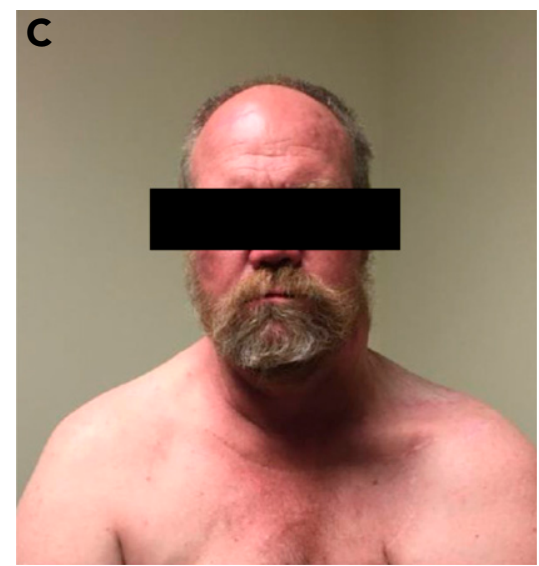

Figure 2. Clinical photographs of the right trapezius mass (A) after vismodegib and phase I therapy, before initiation of pembrolizumab; (B) after 4 pembrolizumab infusions; and (C) after discontinuation of pembrolizumab.

$36.9 \%$ and median PFS of 13.1 months in the metastatic cohort. ${ }^{17,18}$ Sonidegib was FDA-approved in 2015 based on data from BOLT, a phase II trial in 230 patients that showed an ORR of $23.1 \%$ (per investigator review) and median PFS of 13.1 months in the metastatic cohort treated with $200 \mathrm{mg}$ of the agent. ${ }^{19-21}$

Despite these options, resistance remains a problem. Sonidegib and vismodegib remain the sole FDA-approved targeted therapies for advanced BCC, and little evidence is available to support the use of a second hedgehog pathway inhibitor as a second-line treatment option. One trial found that patients whose advanced BCC was resistant to vismodegib had similar resistance to sonidegib; 3 of 9 had stable disease and none experienced an objective response. ${ }^{22}$ Effective treatment options are thus extremely limited for patients whose disease progresses on chemotherapy or a hedgehog inhibitor; furthermore, efficacy of these agents in a dedifferentiated clear cell variant of BCC is unknown. This highlights the need for more relevant data for these subgroups, and large-scale trial participation remains crucial although inefficient, owing to the rarity of metastatic BCC and of the clear cell variant in particular. Given these barriers, clinical experience can provide insight and direction for further approaches, as we report herein. Although our patient did not receive further targeted treatment after progression on vismodegib, he had other potentially targetable genomic alterations, and a combination approach might have produced a more meaningful response than hedgehog pathway inhibition alone. For example, truncated PIK3R1 (R386*) may predict sensitivity to inhibitors of the phosphoinositide 3-kinase (PI3K)/Akt/mTOR pathway, and offlabel use of mTOR inhibitors currently approved for other indications (eg, everolimus in renal cell carcinoma, pancreatic neuroendocrine tumors, and breast cancer; temsirolimus in renal cell carcinoma) may have been a viable option. The splice site mutation in TSC1 (splice site 2392-1G $>$ A) results in loss of function of the hamartin protein and mTOR activation, also making a case for an off-label trial of an mTOR inhibitor. Presence of the $M Y C N$ P44L mutation also raises the question whether inhibition of N-Myc expression or indirect targeting of N-Myc would be valuable; Aurora kinase B and BET inhibitors have shown some activity in $M Y C N$-driven tumors in preclinical models and early-phase trials, although these were in malignancies with $M Y C N$ amplification, and it is unclear whether a similar approach would have been of value in this particular case. Also of interest, ARIDIA alterations are associated with clear cell changes in a variety of other cancers, and the mutation in this case (splice site 2878+2_2878+2delT, truncation exon 1) may be responsible for the phenotype seen. Potential therapeutic targets have been proposed in AIRD1A-mutated cancer, including inhibition of histone methyltransferase EZH2 and targeting of PI3K/AKT signaling, thus offering further avenues for combination approaches in metastatic BCC.

Although targeted combinations have the advantage of select and limited toxicities, this approach often results in the development of resistance. Immune checkpoint inhibitors have been shown to improve survival outcomes in melanoma and a variety of solid tumors, and they could be considered to provide more durable response. A variety of tumor characteristics, including dMMR/MSI-high status ${ }^{23,24}$ and high tumor mutational burden, ${ }^{25,26}$ have been shown to be biomarkers for response or increased survival in patients treated with immunotherapy. In this case, the inactivating MLH-1 
10/28/15 - Progressive disease $\mathrm{s} / \mathrm{p}$ vismodegib

A

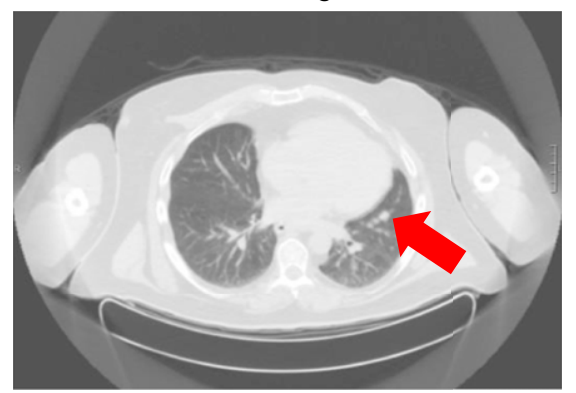

B

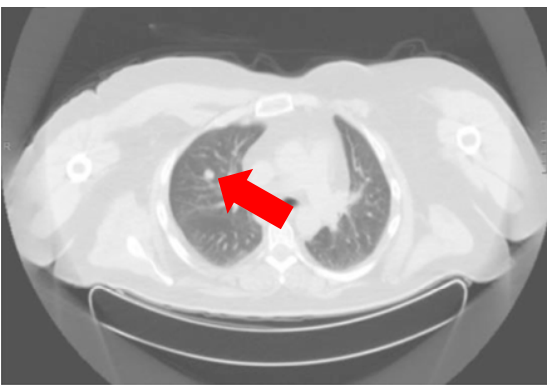

C

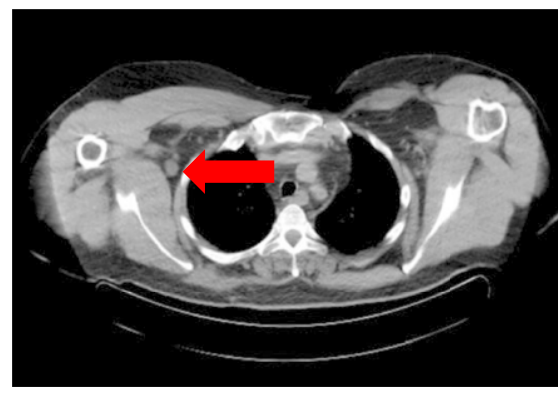

D

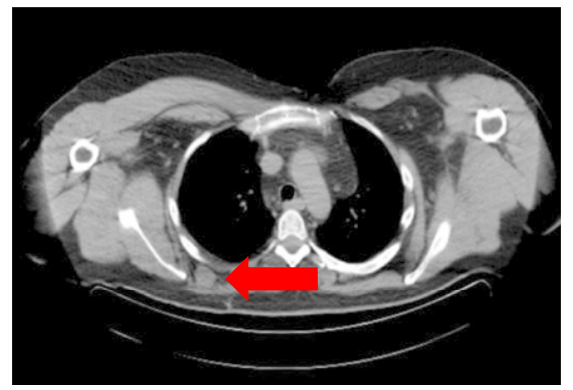

4/14/16 - Progressive disease $s / p$ phase I treatment
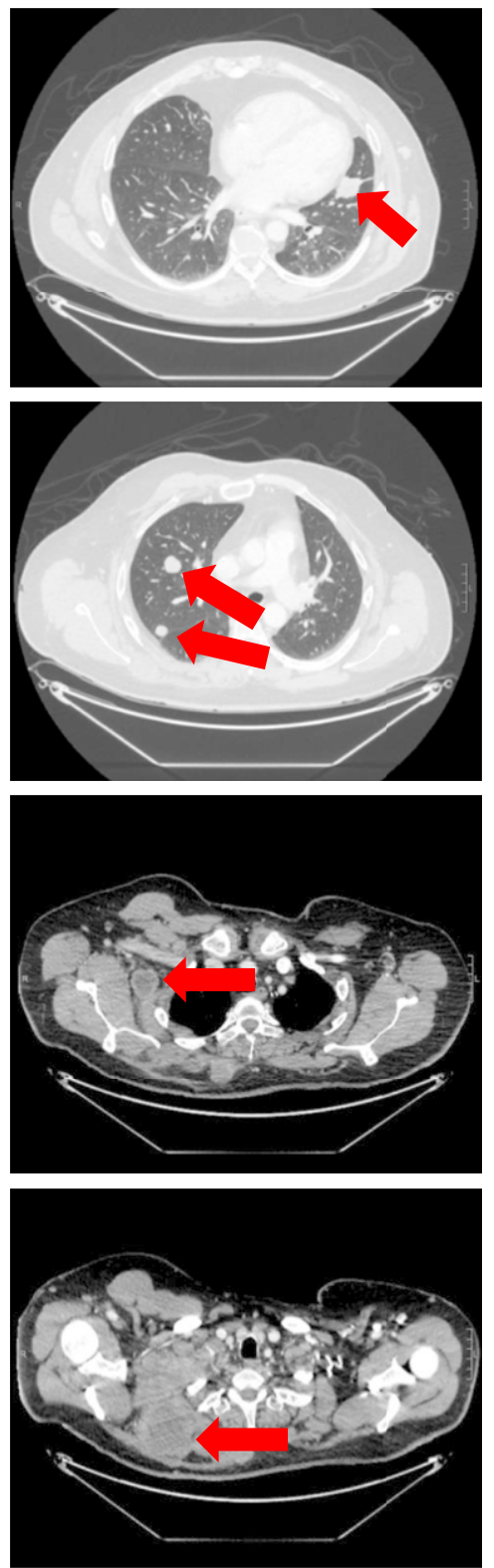

8/1/16 - Disease response s/p 4 pembrolizumab infusions
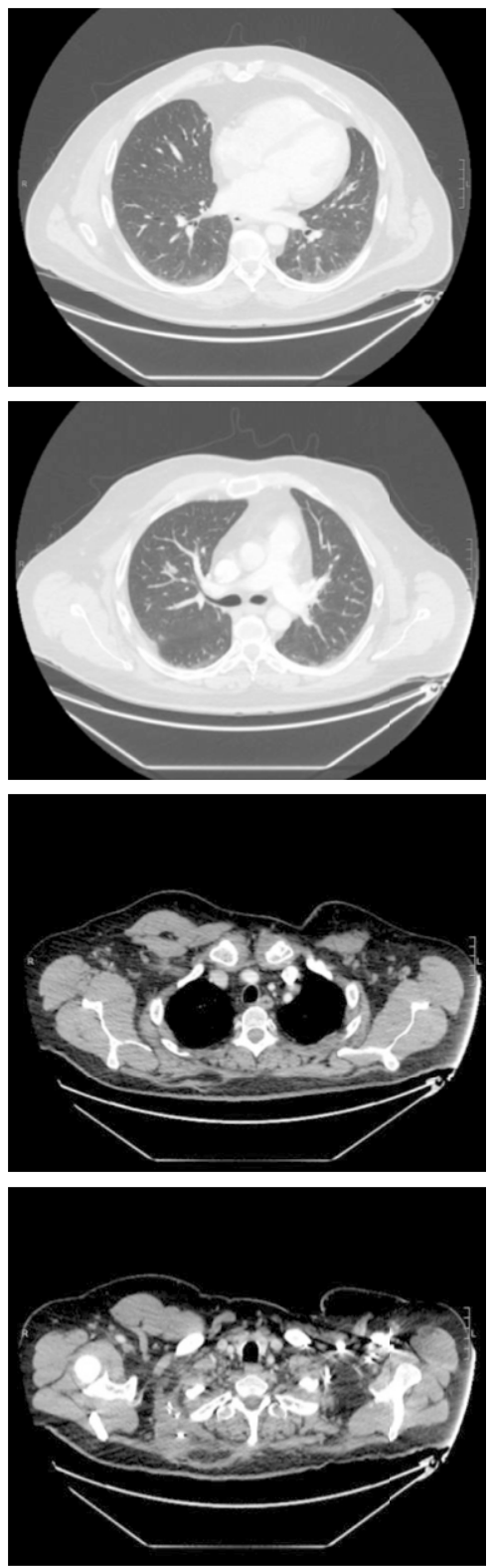

Figure 3. PET/CT images showing metastatic disease (arrows) response in the (A) lingula, (B) right lung, (C) right axillary lymph node, and (D) right trapezius after treatment with vismodegib, phase I therapy, and pembrolizumab.

alteration (as a marker for dMMR/MSI-high) and high number of pathogenic alterations (as a marker of high tumor mutational burden) were compelling grounds on which to offer off-label treatment with an anti-PD-1 agent, despite the PD-L1-negative status of the tumor. An increasing body of nonrandomized data shows immunotherapy to be clinically relevant in the treatment of metastatic BCCs; combination approaches with vismodegib are already being tested (ClinicalTrials.gov identifier: NCT02690948) and monotherapy with anti-PD-1 is under ongoing clinical investigation (NCT03132636). Detailed molecular profiling may provide valuable insight into the tumor microenvironment and identify tumor characteristics in patients who may benefit most from such an approach. For example, recent data from mouse models of ovarian cancer have shown that ARIDIA loss and PIK3CA mutation cooperate to promote carcinogenesis through sustained IL-6 production. ${ }^{27}$ Understanding the landscape of driver mutations and progression pathways in BCC and the mechanisms of resistance to hedgehog pathway 
inhibition is essential for establishing the optimal treatment approach. ${ }^{28}$

\section{Conclusions}

Given the aggressive clinical course and molecular profiling results described in this case, consideration of upfront combination therapies (targeted/targeted or targeted/immunotherapy) may provide a more effective treatment strategy for metastatic BCC, especially in patients exhibiting clear cell change. More widespread use of molecular profiling to identify common aberrant signaling pathways and increased involvement of specialized molecular tumor boards to interpret these results will help inform optimal management of this malignancy.

Submitted June 15, 2018; accepted for publication April 19, 2019

Disclosures: Dr. Tsai has disclosed that she receives grant/research support from OncoSec Medical, Merck, and Bristol Myers-Squibb. Dr. Bastian has disclosed that he is a consultant for Lily Inc. The remaining authors have not received any financial consideration from any person or organization to support the preparation, analysis, results, or discussion of this article.

Correspondence: Katy K. Tsai, MD, Melanoma and Skin Cancer Program, UCSF Helen Diller Family Comprehensive Cancer Center, 1600 Divisadero Street, Box 1770, San Francisco, CA 94115. Email: katy.tsai@ucsf.edu

\section{References}

1. Biswas A, Mahalingam M. Cutaneous clear cell neoplasms: a histopathological reappraisal. Am J Dermatopathol 2012;34:237-254.

2. Smith EH, Andea AA. Cutaneous clear cell neoplasms: a systematic review. Diagn Histopathol 2016;22:134-141.

3. Asgari MM, Moffet HH, Ray GT, et al. Trends in basal cell carcinoma incidence and identification of high-risk subgroups, 1998-2012. JAMA Dermatol 2015;151:976-981.

4. van Domarus MM, Stevens PJ. Metastatic basal cell carcinoma: report of five cases and review of 170 cases in the literature. J Am Acad Dermatol 1984;10:1043-1060.

5. Nguyen-Nielsen $M$, Wang L, Pedersen L, et al. The incidence of metastatic basal cell carcinoma (mBCC) in Denmark, 1997-2010. Eur J Dermatol 2015;25:463-468

6. Snow SN, Sahl W, Lo JS, et al. Metastatic basal cell carcinoma: report of five cases. Cancer 1994;73:328-335.

7. Reifenberger J, Wolter M, Weber RG, et al. Missense mutations in $\mathrm{SMOH}$ in sporadic basal cell carcinomas of the skin and primitive neuroectodermal tumors of the central nervous system. Cancer Res 1998;58:1798-1803.

8. Xie J, Murone M, Luoh SM, et al. Activating smoothened mutations in sporadic basal-cell carcinoma. Nature 1998;391:90-92.

9. Soufir N, Gerard B, Portela M, et al. PTCH mutations and deletions in patients with typical nevoid basal cell carcinoma syndrome and in patients with a suspected genetic predisposition to basal cell carcinoma: a French study. Br J Cancer 2006;95:548-553.

10. Ling G, Ahmadian A, Persson A, et al. PATCHED and p53 gene alterations in sporadic and hereditary basal cell cancer. Oncogene 2001;20:7770-7778.

11. Barr RJ, Williamson C. Clear-cell basal cell carcinoma. Arch Dermatol 1984;120:1086.

12. Fernández-Aceñero MJ, Cenjor C, Córdova S. Clear cell basal cell carcinoma with pulmonary metastasis: case report and literature review. Am J Dermatopathol 2011;33:379-382.

13. Frampton GM, Fichtenholtz A, Otto GA, et al. Development and validation of a clinical cancer genomic profiling test based on massively parallel DNA sequencing. Nat Biotechnol 2013;31:1023-1031.

14. Sekulic A, Migden MR, Oro AE, et al. Efficacy and safety of vismodegib in advanced basal-cell carcinoma. N Engl J Med 2012;366:2171-2179.

15. Sekulic A, Migden MR, Lewis K, et al. Pivotal ERIVANCE basal cell carcinoma (BCC) study: 12-month update of efficacy and safety of vismodegib in advanced BCC. J Am Acad Dermatol 2015;72:1021-1026.e8.

16. Sekulic A, Migden MR, Basset-Seguin N, et al. Long-term safety and efficacy of vismodegib in patients with advanced basal cell carcinoma: final update of the pivotal ERIVANCE BCC study. BMC Cancer 2017;17:332

17. Basset-Séguin N, Hauschild A, Grob JJ, et al. Vismodegib in patients with advanced basal cell carcinoma (STEVIE): a pre-planned interim analysis of an international, open-label trial. Lancet Oncol 2015;16:729-736.

18. Basset-Séguin N, Hauschild A, Kunstfeld R, et al. Vismodegib in patients with advanced basal cell carcinoma: primary analysis of STEVIE, an international, open-label trial. Eur J Cancer 2017;86:334-348.

19. Migden MR, Guminski A, Gutzmer R, et al. Treatment with two different doses of sonidegib in patients with locally advanced or metastatic basal cell carcinoma (BOLT): a multicentre, randomised, double-blind phase 2 trial. Lancet Oncol 2015;16:716-728.

20. Dummer R, Guminski A, Gutzmer R, et al. The 12-month analysis from Basal Cell Carcinoma Outcomes with LDE225 Treatment (BOLT): a phase II, randomized, double-blind study of sonidegib in patients with advanced basal cell carcinoma. J Am Acad Dermatol 2016;75:113-125.e5.

21. Lear JT, Migden MR, Lewis KD, et al. Long-term efficacy and safety of sonidegib in patients with locally advanced and metastatic basal cell carcinoma: 30-month analysis of the randomized phase 2 BOLT study. J Eur Acad Dermatol Venereol 2018;32:372-381.

22. Danial C, Sarin KY, Oro AE, et al. An investigator-initiated open-label trial of sonidegib in advanced basal cell carcinoma patients resistant to vismodegib. Clin Cancer Res 2016;22:1325-1329.

23. Le DT, Uram JN, Wang H, et al. PD-1 blockade in tumors with mismatchrepair deficiency. N Engl J Med 2015;372:2509-2520.

24. Le DT, Durham JN, Smith KN, et al. Mismatch repair deficiency predicts response of solid tumors to PD-1 blockade. Science 2017;357:409-413.

25. Hugo W, Zaretsky JM, Sun L, et al. Genomic and transcriptomic features of response to anti-PD-1 therapy in metastatic melanoma. Cell 2016;165: 35-44.

26. Samstein RM, Lee $\mathrm{CH}$, Shoushtari AN, et al. Tumor mutational load predicts survival after immunotherapy across multiple cancer types. Nat Genet 2019;51:202-206.

27. Chandler RL, Damrauer JS, Raab JR, et al. Coexistent ARID1A-PIK3CA mutations promote ovarian clear-cell tumorigenesis through pro-tumorigenic inflammatory cytokine signalling. Nat Commun 2015;6:6118.

28. Bonilla X, Parmentier L, King B, et al. Genomic analysis identifies new drivers and progression pathways in skin basal cell carcinoma. Nat Genet 2016;48:398-406. 